\title{
Relation between flower colour and pigment composition of tulip (Tulipa L.)
}

\author{
M. NIEUWHOF, J. P. VAN EIJK \& W. EIKELBOOM
}

Institute for Horticultural Plant Breeding, P.O. Box 16, NL 6700 AA Wageningen, Nether.lands

Received 4 January 1989; accepted 23 August 1989

\begin{abstract}
Colour and pigment composition were determined of flowers of $F_{1}$ plants produced by crossing eight tulip cultivars. Yellow flowers only contained carotenoids, orange flowers always at least carotenoids and cyanidin, and red flowers always and pink and purple flowers nearly always cyanidin. Most flowers, except purple ones, did not contain delphinidin. Highest carotenoid levels occurred in yellow, orange, dark red and orange-red flowers; highest delphinidin levels in purple flowers and highest cyanidin and pelargonidin levels in red flowers. Pigment levels in light coloured flowers were lower than in darker coloured flowers. Between flowers with the same colour substantial differences in pigment composition and level may occur, indicating that flower colour of tulip depends on other chemical and physical characters as well.
\end{abstract}

Keywords: Tulipa, tulip, anthocyanidins, carotenoids, cyanidin, delphinidin, flower colour, pelargonidin, pigments

\section{Introduction}

Flower pigment analyses give information on the biochemical background of flower colour and may increase the efficiency of breeding for flower colour in tulips. Flower colour of tulip cultivars seems to be determined by carotenoids and the anthocyanidins delphinidin, cyanidin and pelargonidin (van Eijk et al., 1987). To study the inheritance of these pigments a diallel was made including tulip cultivars differing in pigment composition and flower colour. The inheritance of these pigments was determined largely by additive gene action (Nieuwhof et al., 1988). From the $F_{1}$ plants used for pigment analyses, flower colour also was recorded. As in literature no data on the relation between pigment composition and colour of tulip flowers were found, this subject will be dealt with in this paper.

\section{Materials and methods}

In this study, 1586 plants were included from $44 F_{1}$ populations obtained by cross- 
ing eight tulip cultivars. Contents of carotenoids, delphinidin, cyanidin and pelargonidin in just open flowers of these plants were measured semi-quantitatively and scaled $(0$, trace, 1, 2, 3, 4, 5) as described by van Eijk et al., (1987). For calculation of average pigment levels, traces were estimated as 0.25 . Flower colours were visualized in the middle of the inside of tepals of just open flowers.

Six colour groups were distinguished, white, yellow, orange, red, pink and purple, and within these groups a number of subcolour groups; the main subgroups are mentioned in Table 1.

Table 1. Pigments (composition and level) of tulip flowers with different colours.

\begin{tabular}{|c|c|c|c|c|c|c|c|c|c|c|}
\hline \multirow{2}{*}{$\begin{array}{l}\text { Colour } \\
\text { group }\end{array}$} & \multirow{2}{*}{$\begin{array}{l}\text { Subcolour } \\
\text { group }\end{array}$} & \multirow{2}{*}{$\begin{array}{l}\text { Number } \\
\text { of } \\
\text { plants }\end{array}$} & \multicolumn{2}{|c|}{ Carotenoids } & \multicolumn{2}{|c|}{ Delphinidin } & \multicolumn{2}{|c|}{ Cyanidin } & \multicolumn{2}{|c|}{ Pelargonidin } \\
\hline & & & $\sigma_{0} 1$ & level ${ }^{2}$ & $\%$ & level & $\%$ & level & $\%$ & level \\
\hline \multirow[t]{2}{*}{ Yellow } & yellow & 116 & 100 & 3.1 & 0 & & 0 & & 0 & \\
\hline & light yellow & 35 & 100 & 1.1 & 0 & & 0 & & 0 & \\
\hline \multirow[t]{3}{*}{ Orange } & orange & 21 & 100 & 2.9 & 38 & 0.4 & 100 & 1.4 & 100 & 1.1 \\
\hline & yellow-orange & 10 & 100 & 2.7 & 14 & 0.3 & 100 & 0.8 & 71 & 0.3 \\
\hline & purple-orange & 13 & 100 & 1.9 & 33 & 0.6 & 100 & 1.1 & 80 & 0.8 \\
\hline \multirow[t]{7}{*}{ Red } & red & 98 & 93 & 1.7 & 40 & 0.5 & 100 & 1.9 & 100 & 1.7 \\
\hline & dark red & 10 & 100 & 2.0 & 88 & 0.8 & 100 & 2.2 & 100 & 2.2 \\
\hline & orange-red & 54 & 100 & 2.4 & 18 & 0.6 & 100 & 1.2 & 98 & 1.8 \\
\hline & pink-red & 30 & 93 & 0.7 & 25 & 0.7 & 100 & 1.7 & 90 & 1.1 \\
\hline & purple-red & 88 & 89 & 0.9 & 59 & 1.5 & 100 & 1.9 & 79 & 1.3 \\
\hline & purple-orange-red & 32 & 100 & 1.4 & 38 & 1.0 & 100 & 1.3 & 93 & 0.9 \\
\hline & purple-pink-red & 65 & 87 & 0.7 & 41 & 0.9 & 100 & 1.7 & 95 & 1.0 \\
\hline \multirow[t]{10}{*}{ Pink } & pink & 22 & 77 & 0.6 & 9 & 0.6 & 100 & 1.0 & 59 & 0.8 \\
\hline & light pink & 16 & 68 & 0.4 & 10 & 0.6 & 100 & 0.6 & 15 & 0.5 \\
\hline & orange-pink & 18 & 93 & 1.0 & 6 & 0.3 & 100 & 0.6 & 93 & 0.7 \\
\hline & purple-pink & 277 & 68 & 0.5 & 42 & 0.7 & 99 & 1.1 & 52 & 0.7 \\
\hline & light purple-pink & 184 & 66 & 0.4 & 16 & 0.9 & 98 & 0.7 & 35 & 0.5 \\
\hline & light purple-yellow-pink & 16 & 71 & 1.0 & 14 & 0.6 & 85 & 0.5 & 42 & 0.8 \\
\hline & purple-orange-pink & 77 & 100 & 1.0 & 35 & 0.7 & 98 & 1.1 & 82 & 0.9 \\
\hline & light purple-orange-pink & 21 & 88 & 1.0 & 5 & 0.3 & 94 & 0.8 & 72 & 0.7 \\
\hline & purple-red-pink & 9 & 90 & 0.6 & 20 & 0.4 & 100 & 2.1 & 100 & 0.8 \\
\hline & yellow-purple-pink & 22 & 100 & 1.2 & 52 & 0.9 & 91 & 1.1 & 26 & 0.5 \\
\hline \multirow[t]{4}{*}{ Purple } & purple & 159 & 59 & 0.6 & 97 & 1.6 & 100 & 0.7 & 19 & 0.5 \\
\hline & light purple & 70 & 55 & 0.3 & 97 & 0.8 & 85 & 0.4 & 0 & \\
\hline & yellow-purple & 11 & 100 & 1.3 & 81 & 1.3 & 100 & 0.7 & 18 & 0.3 \\
\hline & pink-purple & 34 & 62 & 0.4 & 97 & 0.9 & 97 & 0.6 & 11 & 0.4 \\
\hline
\end{tabular}

$1 \%$ of plants with pigment.

2 Average level of pigment containing plants. 


\section{Results}

Table 1 gives pigment composition and level of the main subcolour groups $(>8$ plants) and Table 2 the number of plants with specified pigment combinations arranged for different colours.

\section{Pigments related to flower colour}

\section{a. Plants with white flowers}

Flowers of three plants described as yellow-white, contained traces of carotenoids. Plants with completely white flowers (no pigments) were not found.

\section{b. Plants with yellow flowers}

All yellow flowers contained carotenoids, anthocyanidins were generally absent. Flowers of plants of the subgroup yellow contained carotenoids ranging from 1 to 5; light yellow flowers from traces to 2. The carotenoid contents of flowers of four white-yellow plants were scored as trace or 1. A purple-orange-yellow plant contained carotenoids, cyanidin and pelargonidin and another one also delphinidin. A red-yellow plant contained carotenoids and traces of cyanidin and pelargonidin.

Table 2. Numbers of tulip plants with yellow, orange, red, pink, and purple flower colour and specified pigment composition. Car $=$ carotenoids, Del $=$ delphinidin, Cya $=$ cyanidin, Pel $=$ pelargonidin, + $=$ pigment present (classes: trace and 1-5), $-=$ pigment absent (class 0 ).

\begin{tabular}{|c|c|c|c|c|c|c|c|c|c|}
\hline \multicolumn{4}{|c|}{ Pigment composition } & \multirow[t]{2}{*}{ Total } & \multicolumn{5}{|c|}{ Number of plants with colour } \\
\hline Car & Del & Cya & Pel & & yellow & orange & red & pink & purple \\
\hline+ & + & + & + & 251 & 1 & 11 & 138 & 68 & 33 \\
\hline+ & + & + & - & 250 & & 3 & 19 & 87 & 141 \\
\hline+ & + & - & + & 0 & & & & & \\
\hline+ & + & - & - & 14 & & & & 5 & 9 \\
\hline+ & - & + & + & 424 & 2 & 36 & 191 & 193 & 2 \\
\hline+ & - & + & - & 167 & & 3 & 14 & 145 & 5 \\
\hline+ & - & - & + & 2 & & & & 2 & \\
\hline+ & - & - & - & 160 & $158^{1}$ & & & 2 & \\
\hline- & + & + & + & 35 & & & 17 & 14 & 4 \\
\hline - & + & + & - & 115 & & & & 30 & 85 \\
\hline- & + & - & + & 0 & & & & & \\
\hline - & + & - & - & 6 & & & & 2 & 4 \\
\hline- & - & + & + & 85 & & & 12 & 71 & 2 \\
\hline- & - & + & - & 73 & & & 1 & 67 & 5 \\
\hline- & - & - & + & 1 & & & & 1 & \\
\hline- & - & - & - & 3 & & & & 2 & 1 \\
\hline \multicolumn{4}{|c|}{ Total number of plants } & 1586 & 161 & 53 & 392 & 689 & 291 \\
\hline
\end{tabular}

1 Three yellow-white plants included. 


\section{c. Plants with orange flowers}

Orange flowers always contained carotenoids and cyanidin, most also pelargonidin, but were low or without delphinidin. The carotenoid contents in these flowers were usually higher than in flowers with other colours. Between the orange subgroups no important differences in pigments were found.

\section{d. Plants with red flowers}

All red flowers contained cyanidin. The cyanidin contents were usually higher than those of orange, pink and purple flowers. Most red flowers also contained carotenoids, relatively high amounts of pelargonidin, and many also delphinidin. A small number of red flowers only contained carotenoids + cyanidin or cyanidin + pelargonidin. One red plant with only cyanidin was registered.

Between red variants distinct differences in pigment levels were found. High pigment levels were determined in dark red and low levels in pink-red flowers. Highest amounts of carotenoids were measured in orange-red flowers, whereas purple-red flowers had highest delphinidin levels. Pelargonidin contents of most purple variants were low.

\section{e. Plants with pink flowers}

The pink flowering plants is the largest group and showed the largest variation in pigments and tints. Nearly all pink flowers contained cyanidin, most also carotenoids; smaller numbers contained pelargonidin and delphinidin. The levels of these pigments were usually lower than those in orange and red flowers. Most pink flowers contained three pigments, viz. carotenoids, cyanidin and pelargonidin and no delphinidin. Pink flowers with four pigments were found less frequently than with two pigments. Flowers of this last group contained mostly carotenoids + cyanidin or cyanidin + pelargonidin. Many pink flowers contained one pigment only, mostly cyanidin, while in two light yellow-purple-pink plants no pigments could be detected.

Highest carotenoid contents were measured in yellow and orange tinted pink flowers and highest delphinidin and cyanidin contents in purple variants. Most pelargonidin-containing flowers and highest pelargonidin levels were found among orange tinted and purple-red-pink flowers. Pigment levels of 'light' pink variants were generally lower than those of other pink variants.

\section{f. Plants with purple flowers}

Most purple flowers contained cyanidin and delphinidin, many low levels of carotenoids (trace or class 1), and few also pelargonidin at low levels. Most purple flowers contained rather high amounts of delphinidin, while of 15 purple-flowered plants without delphinidin 14 contained cyanidin and four also pelargonidin. Purple flowers with only cyanidin or delphinidin were also detected. In one plant with a light purple flower colour none of the tested pigments was found.

Differences between the purple subgroups were small. In light purple flowers, lower pigment levels were found than in purple flowers and no pelargonidin at all. Yellow-purple flowers contained rather high levels. of carotenoids. 


\section{Combinations of pigments in tulip flowers}

\section{a. Flowers without pigments}

In light purple flowers of one plant and light yellow-purple-pink flowers of two plants no pigments could be detected.

\section{b. Flowers with one pigment}

Flowers with only carotenoids or cyanidin occurred frequently. Flowers with only carotenoids were yellow, flowers of a plant described as light purple-yellow-pink and as light purple-pink also contained only carotenoids (class 1 and trace, respectively). Flowers with only cyanidin were mostly pink, usually with purple tints, five were purple and one purple-red. Flowers of five of the six plants with only delphinidin contained traces of this pigment and had light purplish tints, while flowers of a sixth plant classified in class 1 , were described as pink-purple. Flowers of a plant with only pelargonidin contained a trace of this pigment and were described as light yellow-purple-pink.

\section{c. Flowers with two pigments}

Flowers with carotenoids + cyanidin, delphinidin + cyanidin and cyanidin + pelargonidin were frequently found. Only small numbers of flowers with carotenoids + delphinidin and carotenoids + pelargonidin occurred, and the levels of these pigments were low (trace or class 1). Most of these flowers had light purplish or pink tints. Flowers with only delphinidin + pelargonidin were absent.

\section{d. Flowers with three or four pigments}

Flowers of most plants contained three pigments, mostly carotenoids, cyanidin and pelargonidin or delphinidin, or four pigments. These groups have already been discussed. Flowers containing three anthocyanidins and no carotenoids rather occurred rarely. Such flowers were purplish red when cyanidin concentration was high and purple or purplish pink when it was low.

\section{Discussion}

There was an obvious relation between colour and pigment composition of tulip flowers: yellow flowers contained carotenoids only, orange flowers always carotenoids and cyanidin, red flowers always, pink and purple flowers almost always cyanidin, and most purple flowers delphinidin.

Highest carotenoid levels were found in yellow, orange, dark red and orange-red flowers, highest delphinidin levels in purple and purplish flowers and highest cyanidin and pelargonidin levels in red and reddish flowers. Pigment levels in light and pink coloured flowers were lower than in other colour groups.

Flower colour does not give a sufficient indication of pigments and their levels present in tulip flowers. Between flowers with the same colour differences in pigment composition were found, while flowers with different colours showed the same pigments at equal levels. Discrepancies between pigment composition and flower 
colour may partly be due to chemical variation in anthocyanin composition. In our study the aglycon components (anthocyanidins) were measured and not the sugars with which they form water soluble glycosides (anthocyanins). Shibata \& Ishikura (1960) examined 107 tulip cultivars and identified six anthocyanins, viz. three delphinins, two cyanins and one pelargonin. Halevy \& Asen (1959) found another type of pelargonin. It is likely that chemical variation within anthocyanins contributes to colour variation.

Some variation in the flavonols kaempferol and quercetin as found, may also influence colour expression, directly by yellowing flowers or complexing with anthocyanins (co-pigmentation), giving more blueish tints. Other conditions such as $\mathrm{pH}$ of cell sap, presence of metal ions and physical characteristics of the cells may also affect colour expression (Kamsteeg, 1980). Also variation in composition of carotenoids, localised in chromoplasts, as found in other species (Demuth \& Santamour, 1978; Santamour \& Demuth, 1978) may contribute to flower colour. As flower colour is the ultimate result of a complex of chemical and physical interacting factors a very close correlation between pigments (composition and levels) and flower colour may not be expected.

Van Eijk et al. (1987) and Nieuwhof et al. (1988) ordered tulip flowers with traces of pigments in class 0 . Because a substantial number of flowers was classified as 'trace', it seems more convenient to maintain this as a separate class, as all orange and nearly all pink and purple flowers showed low levels of cyanidin, all orange flowers low levels of pelargonidin, and many pink and purple flowers low levels of carotenoids.

Flowers of three plants in which no pigments were measured were not classified as white, but as light purple or light yellow-purple-pink, indicating that pigment levels of these flowers were below detection limits.

\section{References}

Demuth, P. \& F. S. Santamour Jr., 1978. Carotenoid flower pigments in Liriodendron and Magnolia. Bulletin Torrey Botanical Club 105: 65-66.

Eijk, J. P. van, M. Nieuwhof, H. A. van Keulen \& P. Keijzer, 1987. Flower colour analyses in tulip (Tuli$p a$ L.). The occurrence of carotenoids and flavonoids in tulip tepals. Euphytica 36: 855-862.

Halevy A. H. \& S. Asen, 1959. Identification of the anthocyanins in petals of tulip varieties Smiling Queen and Pride of Haarlem. Plant Physiology 34: 494-499.

Kamsteeg, J., 1980. Genetic control of anthocyanin biosynthesis in Silene dioica. Doctoral Thesis, University of Utrecht, $141 \mathrm{pp}$.

Nieuwhof, M., J. P. van Eijk, P. Keijzer \& W. Eikelboom, 1988. Inheritance of flower pigments in tulip (Tulipa L.). Euphytica 38: 49-55.

Santamour, F. S. Jr. \& P. Demuth, 1978. Carotenoid flower pigments in Rhododendron. HortScience 13: $461-462$.

Shibata, M. \& N. Ishikura, 1960. Paper chromatographic survey of anthocyanin in tulip flowers I. Japanese Journal of Botany 17: 230-238. 